\title{
Entire solutions to sublinear elliptic problems on harmonic $N A$ groups and Euclidean spaces
}

\author{
Ewa Damek $^{1,2} \cdot$ Zeineb Ghardallou ${ }^{1,2}$
}

Received: 18 June 2019 / Accepted: 6 April 2020 / Published online: 30 April 2020

(c) The Author(s) 2020

\begin{abstract}
We give necessary and sufficient conditions for the existence of entire solutions bounded or large of the equation $\mathcal{L} u-p \psi(u)=0$, where $\mathcal{L}$ is either the Laplace operator on $\mathbb{R}^{d}, d \geq 3$ or the Laplace-Beltrami operator on the harmonic $N A$ group and $p$ is a function whose oscillation tends to zero at infinity at a specified rate. The results apply to noncompact rank one symmetric spaces.
\end{abstract}

Keywords Sublinear elliptic problems $\cdot$ Damek-Ricci spaces $\cdot$ Entire solutions $\cdot$ Large solutions

Mathematics Subject Classification Primary: 35JO8 - 35J61 - 53C35 - 58J05 - Secondary: $22 \mathrm{E} 30 \cdot 43 \mathrm{~A} 80 \cdot 53 \mathrm{C} 35$

\section{Introduction}

Let $\mathcal{L}$ be the Laplace operator on $\mathbb{R}^{d}((d \geq 3))$ or the Laplace-Beltrami operator on a harmonic $N A$ group. As a Riemannian manifold such a group is $\mathbb{R}^{d}$ with an appropriate leftinvariant metric. ${ }^{1}$ We are interested in entire continuous solutions to the equation

$$
\mathcal{L} u(x)-\varphi(x, u(x))=0, x \in S,
$$

where $S$ is either $\mathbb{R}^{d}$ or harmonic $N A$, the equation is meant in the sense of distributions and $\varphi: S \times \mathbb{R} \rightarrow \mathbb{R}_{+}$satisfies some appropriate hypotheses. A solution $u$ to (1.1) is called entire if it is defined in the whole space $S$

\footnotetext{
${ }^{1}$ Harmonic NA groups are also called Damek-Ricci spaces. For their basic properties see [1, 4].
}

The first author was supported by the NCN Grant 2019/33/B/ST1/00207.

Zeineb Ghardallou

zeineb.ghardallou@gmail.com

1 Departement of Mathematics, Wroclaw University, Plac Grunwaldzki 2/4, 50-384 Wroclaw, Poland

2 LR11ES11, Department of Mathematical Analysis and Applications, Faculty of sciences of Tunis, University Tunis El Manar, 2092 El Manar, Tunis, Tunisia 
Our aim is to give sufficient necessary and sufficient conditions for the existence of entire solutions bounded or "large". A solution $u$ to (1.1) is called large if $u(x) \rightarrow \infty$ when $d(x, 0) \rightarrow \infty$ where $d$ is the Riemannian distance on $N A$ or the Euclidean distance on $\mathbb{R}^{d}$.

Large solutions, i.e. the boundary blow-up problems are of significant interest due to its various scientific applications in different fields. Such problems arise in the study of Riemannian geometry [3], non-Newtonian fluids [2], the subsonic motion of a gas [13] and the electric potential in some bodies [12].

Throughout this paper, $\varphi$ satisfies the following hypotheses:

$\left(H_{1}\right) \quad$ For every $t_{0} \in[0, \infty), x \mapsto \varphi\left(x, t_{0}\right) \in \mathcal{K}_{d}^{\text {loc }}(S)$, i.e. it is locally in the Kato class in $S$.

$\left(H_{2}\right) \quad$ For every $x_{0} \in S, t \mapsto \varphi\left(x_{0}, t\right)$ is continuous increasing on $[0, \infty)$

$\left(H_{3}\right) \quad \varphi(x, t)=0$ for every $x \in S$ and $t \leq 0$.

Let $\Omega$ be a domain in $S$. We recall that a Borel measurable function $\psi$ on $\Omega$ is locally in the Kato class in $\Omega$ if

$$
\lim _{\alpha \rightarrow 0} \sup _{x \in D} \int_{D \cap(|x-y| \leq \alpha)} \frac{|\psi(y)|}{|x-y|^{d-2}} \mathrm{~d} y=0
$$

for every open bounded set $D, \bar{D} \subset \Omega$. $\left(H_{1}\right)$ makes $\varphi$ locally integrable against the Green function $^{2}$ for $\mathcal{L}$ which plays an important role in our approach.

There is a number of results that indicate that bounded and large solutions cannot occur at the same time. This holds, in particular, in a more general setting of elliptic operators with smooth coefficients, if for every $x, \varphi(x, \cdot)$ is concave as a function of the second variable or $\varphi(x, t)=p(x) \psi(t)$ and $\psi$ is sublinear [5].

In our setting, we are able to say more. Both spaces $\mathbb{R}^{d}$ and harmonic $N A$ have a common feature-global geodesic coordinates and a phenomenon of radiality with respect to the appropriate Riemannian distance $d$. Suppose that, for every $t, \varphi(\cdot, t)$ is radial as a function of $x$, i.e. $\varphi(x, t)=\varphi(d(0, x), t)$, see [6]. Then an entire solution always exists, but if it is bounded or large, it depends on the growth of $\varphi(\cdot, c)$ measured by $I_{\varphi(\cdot, c)}$, where for a radial function $g$ and $r=d(x, 0), I_{g}$ is defined by

$$
I_{g}=\int_{0}^{\infty} r g(r)(r) \mathrm{d} r,
$$

on Euclidean $S$ and

$$
I_{g}=\int_{0}^{\infty} g(r) \mathrm{d} r
$$

on harmonic $S$. To obtain $I_{\varphi(\cdot, c)}$ we fix $c$ and we put $g(r)=\varphi(r, c)$ in (1.2) or (1.3). Then we have the following characterization, see [6]:

Theorem 1 Suppose that $\left(H_{1}\right)-\left(H_{3}\right)$ are satisfied and $\varphi$ is radial with respect to the first variable. Suppose further that either

$\left(H_{4}\right)$ : For every $x_{0} \in S, t \mapsto \varphi\left(x_{0}, t\right)$ is concave on $[0, \infty)$.

or

$\overline{2}$ See (4.1), (4.2) for the definition of the Green function $G_{\Omega}$ in a domain $\Omega$. 
$\left(H_{5}\right): \varphi(x, t)=p(x) \psi(t)$, where $p \in \mathcal{L}_{\mathrm{loc}}^{\infty}(S)$ and there exists a constant $C>0$ such that $\psi(t) \leq C(1+t)$.

Then (1.1) has always an entire radial solution bounded or large. Moreover, there is a nontrivial bounded solution if and only if

$$
\text { there exists } c>0 \text { such that, } I_{\varphi(\cdot, c)}<\infty
$$

and there is a large solution if and only if for every $c>0, I_{\varphi(\cdot, c)}=\infty$. If $\varphi(x, t)=p(x) \psi(t)$ then (1.4) means $I_{p}<\infty$.

In both spaces, in the radial case, $I_{\varphi(\cdot, c)}$ is finite if and only if the Green potential of $\varphi(\cdot, c)$ is well defined. The difference in $I_{\varphi(\cdot, c)}$ for $\mathbb{R}^{d}(1.2)$ and for $N A(1.3)$ is due to the properties of the fundamental solution $G$ to $\mathcal{L}$ on each space. More precisely, in both spaces $G$ is radial. On $\mathbb{R}^{d}, G(x)=a_{d}|x|^{-d+2}$ where $a_{d}$ is a constant depending only on the dimension. For $N A$, we have a precise estimates for $G$ proved in Theorems 21 and 22 in [6].

For $\varphi$ radial with respect to the first variable, Theorem 1 goes considerably beyond the previous results. The aim of this paper is to prove an analogous result for $\varphi(x, t)=p(x) \psi(t)$ and $\left(H_{4}\right)$ or $\left(H_{5}\right)$ satisfied without assuming radiality of $p$. The strategy is to "radialize" $p$ and to make use of Theorem 1. For that we have to control the oscillation of $p$ at infinity, i.e.

$$
p_{\mathrm{osc}}(r)=p^{*}(r)-p_{*}(r)
$$

where

$$
p^{*}(r)=\sup \{p(x): d(x, 0)=r\}
$$

and

$$
p_{*}(r)=\inf \{p(x): d(x, 0)=r\} .
$$

We define a radial function $\rho$ [see (3.5) and (3.6)] that may grow quite fast at infinity and we assume that

$$
I_{\left(\rho p_{\text {osc }}\right)}<\infty
$$

which means that $p_{\text {osc }}$ is "close" at infinity to a radial function. Our result is

Theorem 2 Suppose that $\varphi(s, t)=p(s) \psi(t),\left(H_{2}\right)-\left(H_{3}\right)$ are satisfied, $p^{*} \in \mathcal{K}_{d}^{\text {loc }}(S)$, and (1.5) holds. Suppose further that $\left(H_{4}\right)$ or $\left(H_{5}\right)$ are satisfied. Then

$$
\mathcal{L} u(x)-p(x) \psi(u(x))=0, x \in S,
$$

has always an entire continuous solution. Moreover, there is a nontrivial bounded solution if and only if $I_{p_{*}}<\infty$ and there is a large solution if and only if $I_{p_{*}}=\infty$.

For existence of nontrivial bounded solutions, weaker conditions are sufficient, see Theorem 3. For $p$ radial the above result reduces to Theorem 1 .

For the Laplace operator in $\mathbb{R}^{d}$ and $\varphi(x, t)=p(x) t^{\gamma}$ where $0<\gamma<1$ and $p$ satisfying some additional hypotheses, the problem of entire solutions was considered in $[7,8,11]$. Theorems 2 and 3 not only generalize the previous results to a considerably larger class of nonlinearities but give an analogous characterization for harmonic NA groups.

In particular, the result applies to rank one symmetric spaces and 


$$
\mathcal{L} u(x)-p(x) u(x)^{\gamma}=0, \quad \gamma \leq 1 .
$$

It has a bounded solution if $I_{p_{*}}<\infty$ and a large solution if $I_{p_{*}}=\infty$ provided $I_{\left(\rho p_{\text {osc }}\right)}<\infty$ and, if $p$ is additionally bounded then $\rho$ is polynomially growing or exponentially growing depending whether $\gamma<1$ or $\gamma=1$.

These types of problems have been studied in a more general framework $[5,9,10]$ where $L$ is a second order elliptic operator with $C^{\infty}$ coefficients defined in a Greenian domain $\Omega \subset \mathbb{R}^{d}(d \geq 3)$ such that $L 1 \leq 0$ and $\varphi: \Omega \times \mathbb{R} \rightarrow \mathbb{R}$ be a measurable function. The present paper is based on those results. Some of them are in "Appendix", but for most of them we refer to $[5,9,10]$.

At last, the authors want to express their gratitude to Krzysztof Bogdan, Konrad Kolesko, Mohamed Selmi and Mohamed Sifi for their helpful and kindly suggestions.

\section{Bounded solutions}

Existence of entire nontrivial bounded solutions to the equation (1.1) is guaranteed by essentially weaker assumptions than those of Theorem 2. In particular $\varphi$ does not need to be of the product form. We consider the radial function

$$
\varphi_{\mathrm{osc}}(r, c)=\varphi^{*}(r, c)-\varphi_{*}(r, c),
$$

where

$$
\varphi^{*}(r, c)=\sup \{\varphi(x, c): d(x, 0)=r\}
$$

and

$$
\varphi_{*}(r, c)=\inf \{\varphi(x, c): d(x, 0)=r\}
$$

We have

Theorem 3 (Bounded solution)

Let $\varphi$ satisfies $\left(H_{1}\right)-\left(H_{3}\right)$. We suppose that for every $c>0$

$$
I_{\varphi_{\mathrm{osc}}(\cdot, c)}<\infty,
$$

then there exists a nontrivial nonnegative bounded solution to (1.1) if and only if

$$
\text { there exists } c_{1}>0 \text { such that } I_{\varphi_{*}\left(\cdot, c_{1}\right)}<\infty \text {. }
$$

If $\varphi(x, t)=p(x) \psi(t)$ then $(2.2)$ reduces to $I_{p_{*}}<\infty$.

Proof Suppose that there exists a nonnegative nontrivial bounded solution to (1.1). We have

$$
0 \leq \varphi_{*}(x, c) \leq \varphi(x, c) \text { for every } \mathrm{c}>0 .
$$

By Proposition 16 in [10], there exists a nonnegative nontrivial bounded solution to the equation

$$
\mathcal{L} u=\varphi_{*}(\cdot, u), \text { in } \mathrm{S} .
$$


Consequently, by Theorem 5 in [6], there exists $c_{1}>0$ such that $I_{\varphi_{*}\left(\cdot, c_{1}\right)}<\infty$.

Now let us focus on sufficiency of the condition. We are going to apply Theorem 14, see "Appendix" which is a basic tool in obtaining bounded solutions. It is enough to prove that

$$
G_{S}\left(\varphi\left(\cdot, c_{1}\right)\right)(0)<\infty
$$

where $G_{S}$ is the Green function for $\mathrm{S}$.

Let $G: S \rightarrow \mathbb{R}_{+}$be the fundamental solution for $\mathcal{L}$ given by $G(s)=G_{S}(s, 0), s \in S$. $G$ is radial and for $S=N A$ it satisfies the estimates

$$
c \exp (-Q r) \leq G(r) \leq d \exp (-Q r), \text { for } r \geq 1,
$$

and

$$
G(r) \asymp r^{2-d} \text {, for } 0<r<1,
$$

see [6]. $Q=\frac{p}{2}+q$ is the homogeneous dimension of $N$ [4], i.e $q=\operatorname{dim} \mathcal{Z}, p=\operatorname{dim} \mathcal{V}$, where $\mathcal{N}=\mathcal{Z} \oplus \mathcal{V}$ is the orthogonal decomposition of the Lie algebra of $N$. We define also $G(\cdot, \cdot) \quad$ on $\quad S \times S \quad$ by $\quad G(x, y)=G(x-y)=G_{S}(x-y, 0) \quad$ for $\quad S=\mathbb{R}^{d}, \quad d \geq 3 \quad$ or $G\left(s, s_{1}\right)=G\left(s_{1}^{-1} s\right)=G_{S}\left(s_{1}^{-1} s, 0\right)$ for $S=N A$. Moreover,

$$
G_{S} f(s)=\int_{N A} G\left(s, s_{1}\right) f\left(s_{1}\right) \mathrm{d} m_{L}\left(s_{1}\right),
$$

where $d m_{L}$ is the left Haar measure on S. See [6] for more properties of $G$ on $N A$.

From now we continue the proof for $N A$ groups. For $\mathbb{R}^{d}$, we use estimates of the corresponding fundamental solution and follow the same argument. Suppose that there exists $c_{1}>0$ such that $I_{\varphi_{*}\left(\cdot, c_{1}\right)}<\infty$. Then by (2.1) $I_{\varphi^{*}\left(\cdot, c_{1}\right)}<\infty^{3}$ and so by $(2.5)$

$$
\int_{1}^{+\infty} G(r) \varphi^{*}\left(r, c_{1}\right) 2^{p+q} \sinh \left(\frac{r}{2}\right)^{p+q} \cosh \left(\frac{r}{2}\right)^{q} \mathrm{~d} r<\infty .
$$

Writing $d m_{L}$ in the radial coordinates see Section 3 in [6] we have

$$
\begin{gathered}
\int_{1}^{+\infty} G(r) \varphi^{*}\left(r, c_{1}\right) 2^{p+q} \sinh \left(\frac{r}{2}\right)^{p+q} \cosh \left(\frac{r}{2}\right)^{q} \mathrm{~d} r \\
=\int_{S-B(0,1)} G(0, s) \varphi^{*}\left(s, c_{1}\right) \mathrm{d} m_{L}(s) \\
=\int_{S-B(0,1)} G_{S}(0, s) \varphi^{*}\left(s, c_{1}\right) \mathrm{d} m_{L}(s)
\end{gathered}
$$

Hence

$$
\int_{S-B(0,1)} G_{S}(0, s) \varphi\left(s, c_{1}\right) \mathrm{d} m_{L}(s)<\infty .
$$

By $\left(H_{1}\right)$, the integral on $B(0,1)$ is finite and so we conclude that

\footnotetext{
${ }^{3} \varphi^{*}$ does not necessarily satisfy $\left(H_{1}\right)$ otherwise we could use Proposition 16 in [10] as before.
} 


$$
\int_{S} G_{S}(0, s) \varphi\left(s, c_{1}\right) \mathrm{d} m_{L}(s)<\infty .
$$

\section{Large solutions}

In this section, we prove Theorem 2. To some extent we follow the ideas of [8]. However, now the nonlinearity is more general and we require an additional regularity of $\psi$ to define $\rho$ and to formulate the result precisely. This is done in Proposition 8 and Corollary 10 . Before, we need to develop some preparatory material.

In addition to (1.6) we consider the following equations

$$
\begin{aligned}
& \mathcal{L} u=p_{*} \psi(u), \text { in } S . \\
& \mathcal{L} u=p^{*} \psi(u), \text { in } S .
\end{aligned}
$$

For a radial function $g$, we have

$$
(\mathcal{L} g)(x)=\left(L_{\mathrm{rad}} g\right)(r),
$$

where, for $S$ with multiplicities $p, q$,

$$
\begin{aligned}
L_{\mathrm{rad}} & =\partial_{r}^{2}+\left(\frac{p+q}{2} \operatorname{coth}\left(\frac{r}{2}\right)+\frac{q}{2} \tanh \left(\frac{r}{2}\right)\right) \partial_{r} \\
& =\partial_{r}^{2}+\left(\frac{p}{2} \operatorname{coth}\left(\frac{r}{2}\right)+q \operatorname{coth}(r)\right) \partial_{r}
\end{aligned}
$$

and $r=d(x, 0)$, see, e.g. [1].

Proposition 4 On $S=N A$ we define a function $V(r)=V(r(x))$ by

$$
\begin{aligned}
V(r) & =\int_{0}^{r} \frac{1}{\sinh \left(\frac{s}{2}\right)} \int_{0}^{s} \sinh \left(\frac{t}{2}\right) p_{*}(t) \mathrm{d} t \mathrm{~d} s, \\
& =\int_{0}^{r} \sinh \left(\frac{t}{2}\right) p_{*}(t) \int_{t}^{r} \frac{1}{\sinh \left(\frac{s}{2}\right)} \mathrm{d} s \mathrm{~d} t,
\end{aligned}
$$

$r \geq 0$. Then

$$
\mathcal{L} V=L_{\mathrm{rad}}(V) \geq p_{*} .
$$

Remark 5 Function $V$ is the first ingredient to control oscillation of $p$.

Proof We have 


$$
\begin{aligned}
\left(\partial_{r} V\right)(r) & =\frac{1}{\sinh \left(\frac{r}{2}\right)} \int_{0}^{r} \sinh \left(\frac{t}{2}\right) p_{*}(t) \mathrm{d} t \\
\left(\partial_{r}^{2} V\right)(r) & =p_{*}(r)+\left[\int_{0}^{r} \sinh \left(\frac{t}{2}\right) p_{*}(t) \mathrm{d} t\right]\left(-\frac{1}{2} \frac{\cosh \left(\frac{r}{2}\right)}{\sinh \left(\frac{r}{2}\right)^{2}}\right) \\
& =p_{*}(r)+\left[\int_{0}^{r} \sinh \left(\frac{t}{2}\right) p_{*}(t) \mathrm{d} t\right]\left(-\frac{1}{2} \frac{\operatorname{coth}\left(\frac{r}{2}\right)}{\sinh \left(\frac{r}{2}\right)}\right) \\
& =p_{*}(r)-\frac{1}{2} \operatorname{coth}\left(\frac{r}{2}\right)\left(\partial_{r} V\right)(r) .
\end{aligned}
$$

Therefore, by (3.3) we obtain

$$
\begin{aligned}
\left(L_{\mathrm{rad}} V\right)(r) & =p_{*}(r)-\frac{1}{2} \operatorname{coth}\left(\frac{r}{2}\right)\left(\partial_{r} V\right)(r)+\left(\frac{p}{2} \operatorname{coth}\left(\frac{r}{2}\right)+q \operatorname{coth}(r)\right)\left(\partial_{r} V\right)(r) \\
& =p_{*}(r)+\left(\frac{p-1}{2} \operatorname{coth}\left(\frac{r}{2}\right)+q \operatorname{coth}(r)\right)\left(\partial_{r} V\right)(r) \\
& \geq p_{*}(r) .
\end{aligned}
$$

Remark 6 For $\mathcal{L}=\Delta$ the Laplace operator in the Euclidean space $\mathbb{R}^{d}$, we have the following analogue of Proposition 4. Let

$$
V(r)=\frac{1}{d-2} r^{2} \int_{0}^{1}\left(1-t^{d-2}\right) t p_{*}(r t) \mathrm{d} t, r \geq 0 .
$$

Then

$$
\Delta(V)=p_{*} .
$$

For the equation $\Delta u=p u^{\gamma}$ with $p \in \mathcal{L}_{\text {loc }}^{\infty}\left(\mathbb{R}^{d}\right)$ and $0<\gamma<1, V$ was introduced in [8] and to prove (3.4) El Mabrouk and Hansen use the formula $\partial_{r}^{2}+\frac{d-1}{r} \partial_{r}$ for the radial part of $\Delta$.

Function $\psi$ is the second ingredient to construct $\rho$. In fact, we need to dominate $\psi$ by a more regular function $\psi_{1}$. In [8], for $\psi(t)=t^{\gamma}$, such regularization was not needed.

Lemma 7 Suppose that $\psi$ is a positive function satisfying $\left(H_{2}\right)$ and $\int_{0}^{1} \psi(y) \mathrm{d} y>0$. Let $0<\theta<1$ and let $\eta$ be defined by

$$
\eta: x \mapsto \begin{cases}1, & \text { on }[-1,0] \\ \exp \left(\frac{1}{\theta}\right) \exp \left(\frac{1}{x^{2}-\theta}\right), & \text { on }] 0, \sqrt{\theta}[ \\ \exp \left(\frac{1}{\theta}\right) \exp \left(\frac{1}{(x+1)^{2}-\theta}\right), & \text { on }]-1-\sqrt{\theta},-1[ \\ 0, & \text { otherwise. }\end{cases}
$$


Then $\psi_{1}=\psi * \eta \in \mathcal{C}^{1}(\mathbb{R})$ satisfies:

- $\psi_{1} \geq \psi$ on $\mathbb{R}$.

- $\psi_{1}^{\prime} \geq 0$ on $\mathbb{R}$.

- $\psi_{1}(0)>0$.

\section{Proof Let}

$$
h: x \mapsto \begin{cases}\exp \left(\frac{1}{\theta}\right) \exp \left(\frac{1}{x^{2}-\theta}\right), & \text { on }]-\sqrt{\theta}, \sqrt{\theta}[ \\ 0, & \text { otherwise. }\end{cases}
$$

It is clear that $h$ is $\mathcal{C}^{1}(\mathbb{R})$ then $\eta$ is $\mathcal{C}^{1}(\mathbb{R})$ too. Following this $\psi_{1} \in \mathcal{C}^{1}(\mathbb{R})$ satisfying

$$
\begin{aligned}
\psi_{1}^{\prime}(x) & =\int_{\mathbb{R}} \psi(x-y) \eta^{\prime}(y) \mathrm{d} y \\
& =\int_{[-1-\sqrt{\theta},-1]} \psi(x-y) h^{\prime}(y+1) \mathrm{d} y+\int_{[0, \sqrt{\theta}]} \psi(x-y) h^{\prime}(y) \mathrm{d} y \\
& =\int_{[0, \sqrt{\theta}]} \psi(x+1+s) h^{\prime}(-s) \mathrm{d} s+\int_{[0, \sqrt{\theta}]} \psi(x-y) h^{\prime}(y) \mathrm{d} y
\end{aligned}
$$

Since $h$ is an even function, $h^{\prime}$ is an odd function and

$$
\psi_{1}^{\prime}(x)=\int_{[0, \sqrt{\theta}]}(\psi(x-y)-\psi(x+1+y)) h^{\prime}(y) \mathrm{d} y
$$

in addition, $h^{\prime} \leq 0$ on $[0, \sqrt{\theta}]$ and $\psi(x-y)-\psi(x+1+y) \leq 0$ if $y \in[0, \sqrt{\theta}]$, then

$$
\psi_{1}^{\prime}(x) \geq 0, \text { on } \mathbb{R} \text {. }
$$

Also

$$
\begin{aligned}
\psi_{1}(x)=\int_{\mathbb{R}} \psi(x-y) \eta(y) \mathrm{d} y & \geq \int_{-1}^{0} \psi(x-y) \eta(y) \mathrm{d} y \\
& \geq \int_{-1}^{0} \psi(x-y) \mathrm{d} y \geq \psi(x)
\end{aligned}
$$

In addition,

$$
\begin{aligned}
\psi_{1}(0) & =\int_{\mathbb{R}} \psi(-y) \eta(y) \mathrm{d} y \\
& \geq \int_{-1}^{0} \psi(-y) \eta(y) \mathrm{d} y=\int_{0}^{1} \psi(y) \mathrm{d} y>0 .
\end{aligned}
$$

Let us recall that a continuous function $u$ is a supersolution (subsolution) to (3.1) if $\mathcal{L} u-p_{*} \psi(u) \leq 0\left(\mathcal{L} u-p_{*} \psi(u) \geq 0\right.$, respectively $)$.

Now we are ready to prove that all radial supersolutions of (3.1) having the same value at 0 can be dominated by means of the function $V$. 
Proposition 8 Suppose that $\psi$ satisfies $\left(H_{2}\right)-\left(H_{4}\right)$. Let $V$ and $\psi_{1}$ be as in Proposition 4 and Lemma 7. Let $u$ be a radial supersolution of (3.1) such that $\mathcal{L} u \in L_{\mathrm{loc}}^{1}(S)$ then

$$
u \leq F=\varsigma \circ\left(\varsigma^{-1}(u(0))+V\right), \text { in } S,
$$

where $\varsigma=\mu^{-1}$ and $\mu(r)=\int_{0}^{r} \frac{1}{\psi_{1}(y)} \mathrm{d} y$.

Remark 9 Notice that in view of Lemma 7, $\mu$ and $\varsigma$ are well defined. ${ }^{4}$ For $\psi$ satisfying $\left(H_{2}\right)$ $-\left(H_{4}\right)$, we note

$$
\rho=\psi(F)=\psi\left(\varsigma \circ\left(\varsigma^{-1}(u(0))+V\right)\right) .
$$

If in addition, $\psi$ is $\mathcal{C}^{1}$ integrable at 0 then we can take $\mu(r)=\int_{0}^{r} \frac{1}{\psi(y)} \mathrm{d} y$.

In particular, for $\psi(t)=t^{\gamma}$ for some $0<\gamma<1$ we obtain

$$
u \leq C\left(u(0)^{1-\gamma}+V\right)^{\frac{1}{1-\gamma}}
$$

as in [8].

More generally, notice that $\varsigma$ and $V$ are strictly increasing and so is $F$. The growth of $\psi_{1}$ is the same as the growth of $\psi$ and it is sublinear. So the growth of $\mu$ may be like $\log r$ which makes $\psi \circ \varsigma$ to grow exponentially. If $\psi$ grows like $t^{\gamma}$ then $\psi \circ \varsigma$ grows polynomially. Introducing $F$ as above we cover a much larger range of nonlinearities than in [8].

Proof By the previous lemma, $\mu$ is well defined on $\mathbb{R}_{+}$, it belongs to $\mathcal{C}^{1}\left(\mathbb{R}_{+}\right)$and $\mu^{\prime}(x)=\frac{1}{\psi_{1}(x)}>0$. We denote $\varsigma=\mu^{-1}$ on $[0, \infty)$-the inverse function to $\mu$. Then

- $\varsigma(0)=0, \varsigma(x)>0$ for $x>0$.

- $\varsigma^{\prime}(x)=\psi_{1} \circ \varsigma(x)>0$ for $x \geq 0$.

- $\varsigma^{\prime \prime}(x)=\varsigma^{\prime}(x) \psi_{1}^{\prime}(\varsigma(x)) \geq 0$ for $x \geq 0$.

Let $\epsilon>0, a=\varsigma^{-1}(u(0)+\epsilon)$ and $g(r)=\varsigma(a+V(r))$. Then $g$ is radially symmetric satisfying $g(0)=u(0)+\epsilon>u(0)$ and $g>0$ on $S$. We will do the calculation on NA. For $\Delta$ on $\mathbb{R}^{d}$ it is analogous. In addition,

$$
\begin{aligned}
& \left(\partial_{r} g\right)(r)=\left(\partial_{r} V\right)(r) \varsigma^{\prime}(a+V(r)) \\
& \left(\partial_{r}^{2} g\right)(r)=\left(\partial_{r}^{2} V\right)(r) \varsigma^{\prime}(a+V(r))+\left(\partial_{r} V\right)^{2}(r) \varsigma^{\prime \prime}(a+V(r))
\end{aligned}
$$

then

$$
\begin{aligned}
\left(L_{\mathrm{rad}} g\right)(r) & =\varsigma^{\prime}(a+V(r))\left(L_{\mathrm{rad}} V\right)(r)+\left(\partial_{r} V\right)^{2}(r) \varsigma^{\prime \prime}(a+V(r)) \\
& \geq \varsigma^{\prime}(a+V(r))\left(L_{\mathrm{rad}} V\right)(r) \\
& \geq \varsigma^{\prime}(a+V(r)) p_{*}(r)=\psi_{1}(g(r)) p_{*}(r) \\
& \geq \psi(g(r)) p_{*}(r)
\end{aligned}
$$

Consequently, $g$ is a subsolution of (3.1) and $L_{\mathrm{rad}}(g)$

${ }^{4}$ In view of $\left(H_{2}\right)-\left(H_{4}\right)$, if $\int_{0}^{1} \psi(y) \mathrm{d} y=0$ then $\psi \equiv 0$. 
is continuous. By Lemma 14 of [6] we get

$$
u \leq \varsigma\left(\varsigma^{-1}(u(0)+\epsilon)+V\right) \text {. }
$$

Letting $\epsilon$ tend to zero we get

$$
u \leq \varsigma\left(\varsigma^{-1}(u(0))+V\right)
$$

In fact, the condition $\left(H_{4}\right)$ may be replaced by sublinearity of $\psi$. Given $\psi$ that satisfies $\left(H_{2}\right)-\left(H_{3}\right)$ and there exists a constant $C>0$ such that $\psi(t) \leq C(1+t)$ we can find $\tilde{\psi}$ satisfying $\left(H_{2}\right)-\left(H_{4}\right)$ and such that $\psi \leq \tilde{\psi}$, see [5].

Corollary 10 Suppose that $\psi$ satisfies $\left(H_{2}\right)-\left(H_{3}\right)$ and $\psi(t) \leq C(1+t)$. Let $\tilde{\psi}$ be a function satisfying $\left(\mathrm{H}_{2}\right)-\left(\mathrm{H}_{4}\right)$ and such that $\psi \leq \tilde{\psi}$. Let $\tilde{F}$ the function constructed in Proposition 8 but for $\tilde{\psi}$. Let $u$ be a radial supersolution of (3.1) such that $\mathcal{L} u \in L_{\mathrm{loc}}^{1}(S)$ then

$$
u \leq \tilde{F}=\tilde{\varsigma} \circ\left(\tilde{\zeta}^{-1}(u(0))+V\right), \text { in } S .
$$

Proof Let $\tilde{\zeta}$ and $\tilde{g}$ be as in the proof of Proposition 8 but defined for $\tilde{\psi}$. Then

$$
\mathcal{L} u-p_{*} \tilde{\Psi}(u) \leq \mathcal{L} u-p_{*} \psi(u) \leq 0 \leq \mathcal{L} g-p_{*} \tilde{\Psi}(g) .
$$

As before, we may apply Lemma 14 of [6] and we get

$$
u \leq \tilde{\varsigma}\left(\tilde{\varsigma}^{-1}(u(0)+\epsilon)+V\right) .
$$

Letting $\epsilon$ tend to zero we get

$$
u \leq \tilde{\varsigma}\left(\tilde{\varsigma}^{-1}(u(0))+V\right)=\tilde{F}
$$

Remark 11 Hence for $\psi$ satisfying $\left(H_{2}\right)-\left(H_{3}\right)$ and $\psi(t) \leq C(t+1)$ we note

$$
\rho=\psi(\tilde{F})=\psi\left(\tilde{\zeta}\left(\tilde{\zeta}^{-1}(u(0))+V\right)\right)
$$

Now we are ready to formulate our main result. Later on

$$
\rho= \begin{cases}\psi(F), & \text { if }\left(H_{4}\right) \text { is satisfied, see (3.5), } \\ \psi(\tilde{F}), & \text { if }\left(H_{5}\right) \text { is satisfied, see (3.6). }\end{cases}
$$

Theorem 12 (Large solution) We suppose that $\varphi(s, t)=p(s) \psi(t),\left(H_{1}\right)-\left(H_{3}\right)$ are satisfied and $p^{*} \in \mathcal{K}_{d}^{\text {loc }}(S)$. We assume in addition that $\left(H_{4}\right)$ or $\left(H_{5}\right)$ is satisfied and

$$
I_{\left(\rho p_{\text {osc }}\right)}<\infty
$$

Then (1.1) has a large solution in $S$ if and only if

$$
I_{p_{*}}=\infty
$$


Remark 13 Notice that $V$ defined as in Remark 6 is dominated by $(d-2)^{-1} \int_{0}^{r} t p_{*}(t) \mathrm{d} t$ so we may take

$$
\rho=\psi\left(\left(1+\int_{0}^{r} t p_{*}(t) \mathrm{d} t\right)^{1 /(1-\gamma)}\right)
$$

as it was done in [8].

Equation (3.7) means that $p_{\text {osc }}$ must decay quite fast at $\infty$ and the rate of decay depends both on $\psi$ and $p_{*}$. Suppose that $p_{*}$ is bounded and not integrable (at infinity). Then by (2.5) and the formula for $m_{L}, I_{p_{*}}=\infty$. Then $V(r)$ basically grows linearly. If $\psi$ is linear at $\infty$, then $\psi \circ \varsigma$ or $\psi \circ \tilde{\varsigma}$ grows exponentially; if $\psi(t)=t^{\gamma}$, then $\psi \circ \varsigma$ grows polynomially. The same applies to $\rho$ which determines the behavior of $p_{\text {osc }}$ at $\infty$. If $p_{*}$ is unbounded $V(r)$ may grow faster than linearly and $\rho$ faster than exponentially.

Proof Since $F \geq 1$ ( resp. $\tilde{F} \geq 1$ ), necessity of condition follows from Theorem 3 . Indeed, suppose that there is a large solution and $I_{p_{*}}<\infty$. Then by Theorem 3 there is a bounded solution to (1.1). But this is in contradiction with Theorem 2 (resp. Theorem 1) in [5]. So let us focus on sufficiency. Suppose that $I_{p_{*}}=\infty$. By Theorem 1, there exists a large radial solution $v$ to (3.1) satisfying $v(0)=1^{5}$. Moreover,

$$
\mathcal{L} v-p^{*} \psi(v) \leq \mathcal{L} v-p_{*} \psi(v)=0, \text { in } S,
$$

so $v$ is a radial supersolution to Eq. (3.2). Let

$$
D_{n}=\{x \in S, d(x, 0)=n\} .
$$

By Theorem 14, we define a sequence of functions $\left(u_{n}\right)$ by

$$
u_{n}=U_{D_{n}}^{p^{*}} v, n \in \mathbb{N} \text {. }
$$

, i.e. $U_{D_{n}}^{p^{*}} v$ is a radial solution of Eq. (3.2) in $D_{n}$ having the boundary values $v$.

Consequently, by Lemma $15,\left(u_{n}\right)$ is a positive decreasing sequence bounded above by $v$. Let $u=\lim _{n \rightarrow+\infty} u_{n}$. By Lemma 8 in [10], u is a radial solution of (3.2) satisfying $u \leq v$. Furthermore

$$
\mathcal{L} u-p \psi(u) \geq \mathcal{L} u-p^{*} \psi(u)=0, \text { in } S,
$$

and

$$
\mathcal{L} v-p \psi(v) \leq \mathcal{L} v-p_{*} \psi(v)=0, \text { in } S,
$$

, i.e. $u$ is a subsolution to (1.1) and $v$ is a supersolution to (1.1). We argue that there is a solution $w$ to (1.1) such that

$$
u \leq w \leq v, \text { in } S .
$$

Indeed, Let $w_{n}=U_{D_{n}}^{p} v$. Due to Lemma $15,\left(w_{n}\right)$ is a positive decreasing sequence satisfying $u \leq w_{n} \leq v$. Hence again by Lemma 8 in [10], $w=\lim _{n \rightarrow+\infty} w_{n}$ is a solution to (1.1). ${ }^{5}$ If $I_{p_{*}}=\infty$, for any $\alpha \in \mathbb{R}_{+}^{*}$, we can get a large solution $v$ to $\mathcal{L} u-p_{*} \psi(u)=0$ such that $v(0)=\alpha$, see
Theorem 8 in [6]. 
Now, we need to prove that $w$ is a large solution. Actually, it is enough to prove that

$$
\lim _{d(x, 0) \rightarrow+\infty} u(x)=+\infty .
$$

However, $I_{p_{*}}=\infty \Rightarrow I_{p^{*}}=\infty$, then by Theorem $1, u$ is either trivial or unbounded. Moreover, $u$ is $\mathcal{L}$-subharmonic radial in $S$, hence, by the maximum principle for elliptic operators, it follows that

$$
u(x) \leq u\left(x_{0}\right), \quad \text { if } \quad d(x, 0) \leq d\left(x_{0}, 0\right) .
$$

hence $u$ is either trivial or (3.8) is satisfied. It remains to prove that $u$ is nontrivial. By Theorem 14, we have in $D_{n}$

$$
\begin{aligned}
u_{n}(0)+G_{D_{n}}\left(p^{*} \psi\left(u_{n}\right)\right)(0) & =H_{D_{n}} v(0) \\
& =v(0)+G_{D_{n}}\left(p_{*} \psi(v)\right)(0) .
\end{aligned}
$$

Though $v(0)=1$ then in $D_{n}$

$$
u_{n}(0)+G_{D_{n}}\left(p_{\mathrm{osc}} \psi\left(u_{n}\right)\right)(0)=1+G_{D_{n}}\left(p_{*}\left(\psi(v)-\psi\left(u_{n}\right)\right)\right)(0) .
$$

Additionally, $u_{n} \leq v$ in $D_{n}$ so

$$
u_{n}(0)+G_{D_{n}}\left(p_{\mathrm{osc}} \psi\left(u_{n}\right)\right)(0) \geq 1 \text {, in } D_{n} .
$$

In view of Proposition 8 (resp. Proposition 10) applied to $v$, we have ${ }^{6}$

$$
\begin{aligned}
G_{D_{n}}(0, s) p_{\mathrm{osc}}(s) \psi\left(u_{n}\right)(s) & \leq G_{S}(0, s) p_{\mathrm{osc}}(s) \psi(v)(s) \\
& \leq G(0, s) p_{\mathrm{osc}}(s) \rho(r(s))
\end{aligned}
$$

Moreover, writing the left Haar measure in radial coordinates, we have

$$
\begin{aligned}
& \int_{N A} G(0, s) p_{\mathrm{osc}}(s) \rho(r(s)) \mathrm{d} m_{L}(s) \\
& \quad=\int_{0}^{+\infty} G(r) p_{\mathrm{osc}}(r) \rho(r) 2^{p+q} \sinh ^{p+q}\left(\frac{r}{2}\right) \cosh ^{q}\left(\frac{r}{2}\right) \mathrm{d} r .
\end{aligned}
$$

Since $G(r) \sinh ^{p+q}\left(\frac{r}{2}\right) \cosh ^{q}\left(\frac{r}{2}\right)$, is bounded on $\mathbb{R}_{+}$then by (3.7)

$$
\int_{0}^{+\infty} G(r) p_{\mathrm{osc}}(r) \rho(r) 2^{p+q} \sinh ^{p+q}\left(\frac{r}{2}\right) \cosh ^{q}\left(\frac{r}{2}\right) \mathrm{d} r<\infty .
$$

We conclude that

$$
\int_{N A} G_{S}(0, s) p_{\text {osc }}(s) \psi(v)(s) \mathrm{d} m_{L}(s)<\infty,
$$

Letting $n \rightarrow \infty$ in (3.10), by the dominated convergence theorem we have

$$
u(0)+G\left(p_{\mathrm{osc}} \psi(u)\right)(0) \geq 1 .
$$

And so $u$ is not trivial.

\footnotetext{
${ }^{6}$ From now we continue the proof in $N A$ groups. For $\mathbb{R}^{d}$, we use estimates of the corresponding fundamental solution and follow the same argument.
} 
Open Access This article is licensed under a Creative Commons Attribution 4.0 International License, which permits use, sharing, adaptation, distribution and reproduction in any medium or format, as long as you give appropriate credit to the original author(s) and the source, provide a link to the Creative Commons licence, and indicate if changes were made. The images or other third party material in this article are included in the article's Creative Commons licence, unless indicated otherwise in a credit line to the material. If material is not included in the article's Creative Commons licence and your intended use is not permitted by statutory regulation or exceeds the permitted use, you will need to obtain permission directly from the copyright holder. To view a copy of this licence, visit http://creativecommons.org/licenses/by/4.0/.

\section{Appendix}

For the readers convenience we recall two basic facts about elliptic operators in a Greenian domains that have been used in the paper. For the rest of the material we refer to $[5,6,9$, $10]$.

Let $L$ is a second order elliptic operator with $C^{\infty}$ coefficients defined in an open domain $\Omega \subset \mathbb{R}^{d}(d \geq 3)$ such that $L 1 \leq 0$. We say that $\Omega$ is Greenian if there is a function $G_{\Omega}(x, y)$ smooth on $\Omega \times \Omega \backslash\{(x, x): x \in \Omega\}$ such that for every $y \in \Omega$

$$
L G_{\Omega}(\cdot, y)=-\delta_{y}, \quad \text { in the sense of distributions, }
$$

and

$$
G_{\Omega}(\cdot, y), \quad \text { is a potential, }
$$

, i.e. every positive $L$-harmonic function $h$ such that $h(x) \leq G_{\Omega}(x, y)$ is equal 0 . We write

$$
G_{\Omega}(\varphi(\cdot, c))(x)=\int_{\Omega} G_{\Omega}(x, y) \varphi(y, c) \mathrm{d} y .
$$

We have the following characterization of bounded solutions to

$$
L u(x)-\varphi(x, u(x))=0
$$

Theorem 14 Suppose that $L 1 \leq 0$ and $\varphi$ satisfies $\left(H_{1}\right),\left(H_{2}\right)$ and $\left(H_{3}\right)$. Assume further that $\Omega \subset \mathbb{R}^{d}(d \geq 3)$ and there is $c>0$ such that $G_{\Omega}(\varphi(\cdot, c))$ is finite at least on one point ${ }^{7}$. Then positive continuous solutions to (4.3) bounded by $c$ are in one-to-one correspondence with positive L-harmonic functions bounded by $c$ given by

$$
h=u+G_{\Omega}(\varphi(\cdot, u)), \text { in } \Omega .
$$

For a bounded regular domain $D$ satisfying $\bar{D} \subset \Omega$ and $f \in \mathcal{C}^{+}(\partial D)$ (4.4) becomes

$$
H_{D} f=u+G_{D}(\varphi(\cdot, u)) \text {, in } D .
$$

where $H_{D} f$ is the L-harmonic function in D having $f$ as its boundary values. ${ }^{8}$

In what follows the solution $u$ to (4.3) in a bounded regular domain $D$ taking the values of $f$ at the boundary is denoted $u=U_{D}^{\varphi} f$.

\footnotetext{
7 Then $G_{\Omega}(\varphi(\cdot, c))$ is finite at any point because $\varphi$ is locally in the Kato class.

${ }^{8}$ In a bounded regular domain $D\left(H_{1}\right)$ implies that $G_{D}(\varphi(\cdot, c))$ vanishes on $\partial D$.
} 
We need also a Lemma that gives comparison between sub-solutions and super-solutions to (4.3) in $D$. It holds in a considerable generality: we require only that the function $\varphi: D \times \mathbb{R} \mapsto \mathbb{R}$ is increasing with respect to the second variable, see [10]:

Lemma 15 (Comparison with values on the boundary) Let $u, v \in \mathcal{C}(D), L u, L v \in \mathcal{L}_{\text {loc }}^{1}(D)$ and let $\varphi: D \times \mathbb{R} \rightarrow \mathbb{R}$ be an increasing function with respect to the second variable. If

$$
\left\{\begin{array}{l}
\operatorname{Lu}-\varphi(\cdot, u) \leq L v-\varphi(\cdot, v), \\
\liminf _{\substack{x \rightarrow y \\
y \in \partial D}}(u-v)(x) \geq 0
\end{array}\right.
$$

Then:

$$
u-v \geq 0 \text { in } D \text {. }
$$

\section{References}

1. Anker, J.P., Damek, E., Yacoub, C.: Spherical analysis of harmonic $A N$ groups, Estratto dagli Annali della Scuola Normale Superiore di Pisa, Scienze Fisiche e Matematiche-Serie IV, vol. XXIII. Fasc. 4 (1996)

2. Astarita, G., Marrucci, G.: Principles of non-Newtonian fluid mechanics. McGraw-Hill, New York (1974)

3. Cheng, K.-S., Ni, W.-M.: On the structure of the conformal scalar curvature equation on $\mathbb{R}^{n}$. Indiana Univ. Math. J. 41(1), 261-278 (1992)

4. Damek, E., Ricci, F.: Harmonic analysis on solvable extensions of H-type groups. J. Geom. Anal. 2, 213-248 (1992)

5. Damek, E., Ghardallou, Z.: Large versus bounded solutions to sublinear elliptic problems. Bull. Pol. Acad. Sci. Math. 67, 69-82 (2019)

6. Damek, E., Ghardallou, Z.: Sublinear elliptic problems under radiality. Harmonic NA groups and Euclidean spaces. Forum Math. 31(4), 1007-1026 (2019)

7. El Mabrouk, K.: Entire bounded solutions for a class of sublinear elliptic equations. Nonlinear Anal. 58(1-2), 205-218 (2004)

8. El Mabrouk, K., Hansen, W.: Nonradial large solutions of sublinear elliptic problems. J. Math. Anal. Appl. 330, 1025-1041 (2007)

9. Ghardallou, Z.: Positive solution to a nonlinear elliptic problem. Potential Anal. 44(3), 449-472 (2016)

10. Ghardallou, Z.: Positive solutions to sublinear elliptic problem. Colloq. Math. 155, 107-125 (2019)

11. Lair, A.V., Wood, A.W.: Large solutions of sublinear elliptic equations. Nonlinear Anal. 39, 745-753 (2000)

12. Lazer, A.C., McKenna, P.J.: On a problem of Bieberbach and Rademacher. Nonlinear Anal. 21, 327335 (1993)

13. Pohozhaev, S.L.: The Dirichlet problem for the equation $\Delta u=u^{2}$. Sov. Math. Dokl. 1, 1143-1146 (1960)

Publisher's Note Springer Nature remains neutral with regard to jurisdictional claims in published maps and institutional affiliations. 